\title{
The CBM Experiment at FAIR
}

\author{
Volker Friese ${ }^{1}$ \\ Gesellschaft für Schwerionenforschung $\mathrm{mbH}$ \\ Planckstraße 1, 64291 Darmstadt, Germany \\ E-mail:v.friese@gsi.de
}

\section{for the CBM Collaboration}

The CBM experiment will investigate heavy-ion collisions at beam energies from 8 to $45 \mathrm{AGeV}$ at the future accelerator facility FAIR from 2015 on. The goal is to study the QCD phase diagram in the region of moderate temperatures and highest net-baryon densities in search of the first-order phase transition from confined to deconfined matter and the QCD critical point. CBM will cover hadronic, leptonic and photonic observables at extreme interaction rates, thus having access to rarest probes like charm near threshold. We will present the detector concept, results of feasibility studies and the status of preparations.

Critical Point and Onset of Deconfinement - 4th International Workshop GSI Darmstadt, Germany

9-13 July, 2007

\footnotetext{
$1 \quad$ Speaker
} 


\section{Introduction}

It is believed that above a certain critical energy density, strongly interacting matter exists in a deconfined state of quarks and gluons. Such a state can be reached by heating and/or compressing nuclear matter. In accelerator laboratories, this is done by colliding heavy nuclei, thus creating a dense and hot fireball, albeit only in a very limited space-time domain.

Collisions at the highest available energies, like at the RHIC or - soon - at the LHC, lead to an extremely hot but almost net-baryon free reaction zone. In our contemporary view of the QCD phase diagram, driven by lattice QCD results [1], the transition from confined to deconfined matter in this region is a smooth cross-over, while at moderate temperatures but higher densities as accessible with heavy-ion reactions at lower beam energies, a first-order phase transition is expected. Indeed, results from the NA49 experiment at the CERN-SPS indicate that a phase transition sets in at around $30 \mathrm{AGeV}$ beam energy [2].

Both regions of the deconfinement transition are separated by a critical point, the location of which still being subject to large theoretical uncertainties. However, recent results suggest that it might be reachable within the lower SPS beam energy range [3].

The experimental confirmation of both the first-order phase transition and the critical point would be a breakthrough in our understanding of the properties of strongly interacting matter. This motivates the renewed interest in heavy-ion collisions at moderate beam energies as documented in this conference and expressed in the corresponding experimental activities at BNL [4, 5], CERN [6], JINR [7] and FAIR/GSI.

\section{The CBM experiment at FAIR}

The FAIR facility at GSI [8] will offer unique possibilities for the investigation of the QCD phase diagram at extreme net-baryon densities, besides serving a variety of other fields of physics with anti-proton beams for hadron physics, radioactive beams for nuclear structure physics, and highly pulsed ion beams for plasma physics. For the nuclear collision programme, a synchrotron with $300 \mathrm{Tm}$ bending power (SIS-300) will deliver fully stripped external heavyion beams up to uranium with intensities of up to $2 \cdot 10^{9}$ per second at beam energies from 8 to $35 \mathrm{AGeV}$. Lighter ions $(\mathrm{Z} / \mathrm{A}=0.5)$ can be accelerated up to $45 \mathrm{AGeV}$, while proton beams will be available up to $90 \mathrm{GeV}$. The unprecedented beam intensities will allow studying extremely rare probes with high precision but also constitute a high challenge for detectors and electronics.

The CBM (Compressed Baryonic Matter) experiment [9] will be a next-generation fixedtarget detector to be operated at the FAIR heavy-ion synchrotron SIS-300. It is designed to measure hadronic, leptonic and photonic probes in a large acceptance and at the extreme interaction rates offered by the accelerator. CBM aims at a systematic investigation of $\mathrm{A}+\mathrm{A}$, $p+A$ and $p+p$ collisions, in terms of collision energy $\left({\sqrt{s_{N N}}}=4.5-9.3 \mathrm{GeV}\right.$ for heavy nuclei) and system size, with high precision and statistics. In contrast to the low-energy programmes at the RHIC and the SPS, which due to low collision rates will focus on bulk particle production, 

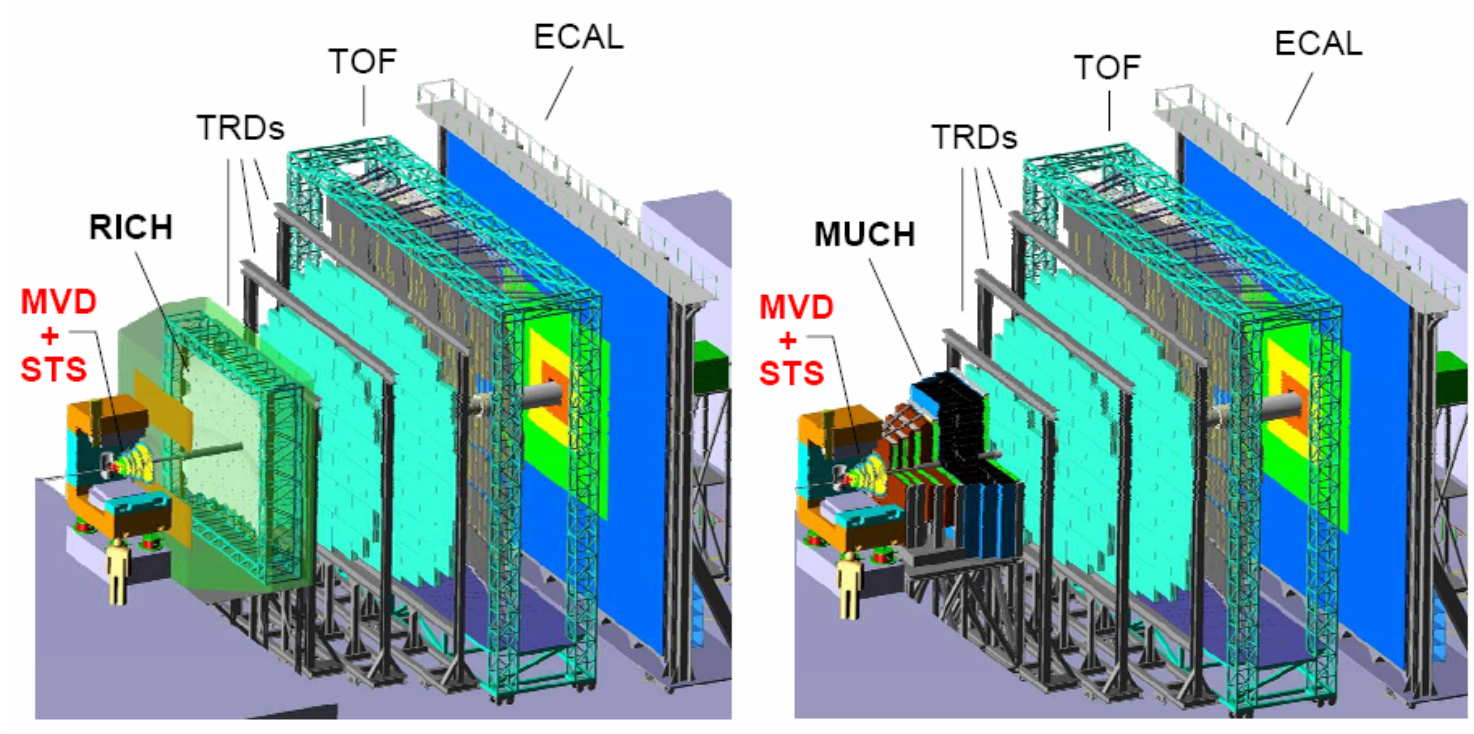

Figure 1. Schematic layout of the CBM experiment. Left: configuration for electron measurements; right: configuration for muon measurements.

CBM will put special emphasis on the measurement of extremely rare probes which have not been accessible by previous heavy-ion experiments at the AGS and the SPS.

The observables to be covered by CBM include multiplicities, phase space distributions and flow of strange and multi-strange hadrons $(\mathrm{K}, \varphi, \Lambda, \Xi, \Omega)$ and charmed hadrons $\left(\mathrm{D}, \mathrm{D}_{\mathrm{s}}, \Lambda_{\mathrm{c}}\right)$. The in-medium properties of short-lived vector mesons $(\varphi, \omega, \varphi)$ will be investigated via their di-leptonic decay. The measurement of lepton pairs will also give access to charmonium states which, together with the open charm measurements, will allow a comprehensive study of charm production near threshold. Signatures of the critical point will be looked for in event-by-event fluctuations of quantities like particle yield ratios, charged multiplicity or average $\mathrm{p}_{\mathrm{t}}$.

To cope with these requirements, the CBM detector must provide hadron and lepton identification in a large acceptance as well as excellent vertexing for the open charm measurements. In addition, the extreme interaction rates needed for the rarest probes call for fast and radiation-hard detectors and read-out electronics, as well as for efficient online data processing and event selection. Currently, two configurations are being evaluated, corresponding to the measurements of electrons and muons, respectively (figure 1). The electron setup comprises a silicon tracking system (STS) inside a dipole magnet, combined with a micro-vertex detector (MVD). Electrons are identified in a ring imaging Cherenkov detector (RICH) and an array of transition radiation detectors (TRD). The setup is completed by a timeof-flight system for hadron identification and an electro-magnetic calorimeter for the detection of neutral particles. For muon measurements, the RICH detector will be replaced by an active absorber system (MUCH).

In the next sections, the detector sub-systems will be discussed along with their performance for the relevant physics observables. All feasibility studies are based on fully reconstructed events using a semi-realistic detector response simulation. The input for the hadronic background was generated with the UrQMD model [10]. 


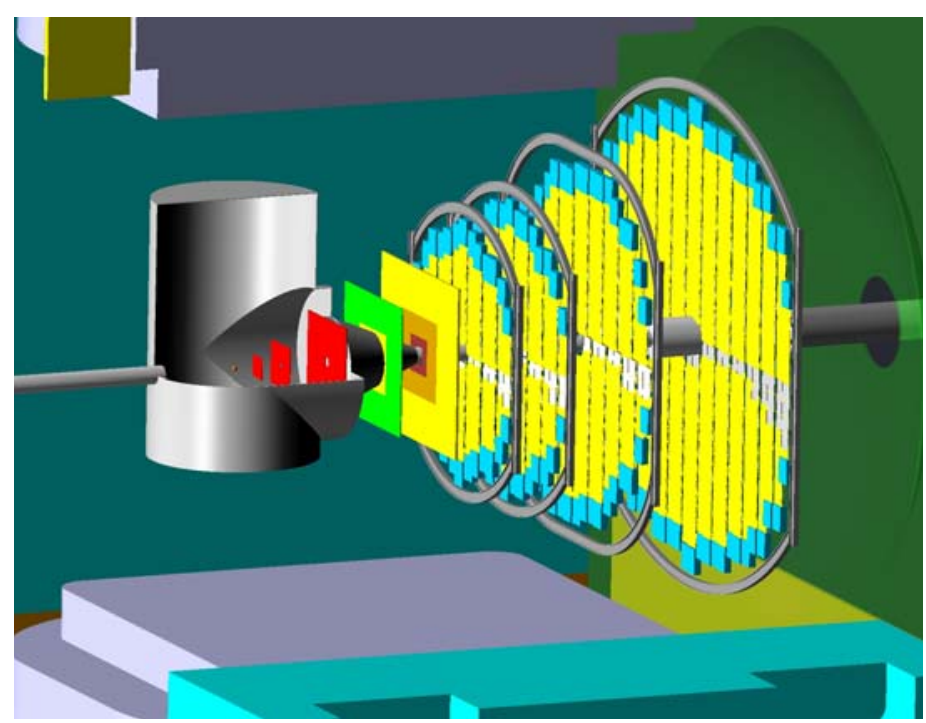

Figure 2. Layout of STS and MVD

\section{STS - the backbone of CBM}

Track reconstruction and momentum measurement in CBM will be performed by a system of silicon tracking stations located in the aperture of a dipole magnet of some $1 \mathrm{Tm}$ bending power. The stations will be built of thin, double-sided strip detectors read out at the top and bottom side outside of the acceptance. A schematic view of the tracking system is shown in figure 2. First sensor prototypes have been developed and produced in summer 2007. In addition, a fast, self-triggered readout chip is being developed. In regions of highest track densities (close to the target and beam), the use of pixel detectors is being considered.

Track reconstruction routines have to cope with the large fake hit rates induced by the projective strip geometry. Solutions based on the Cellular Automaton method and the Hough Transform have already been developed and tested on simulated data. Figure 3 shows the track reconstruction efficiency obtained with the $\mathrm{CA}$ track finder for simulated central $\mathrm{Au}+\mathrm{Au}$ events
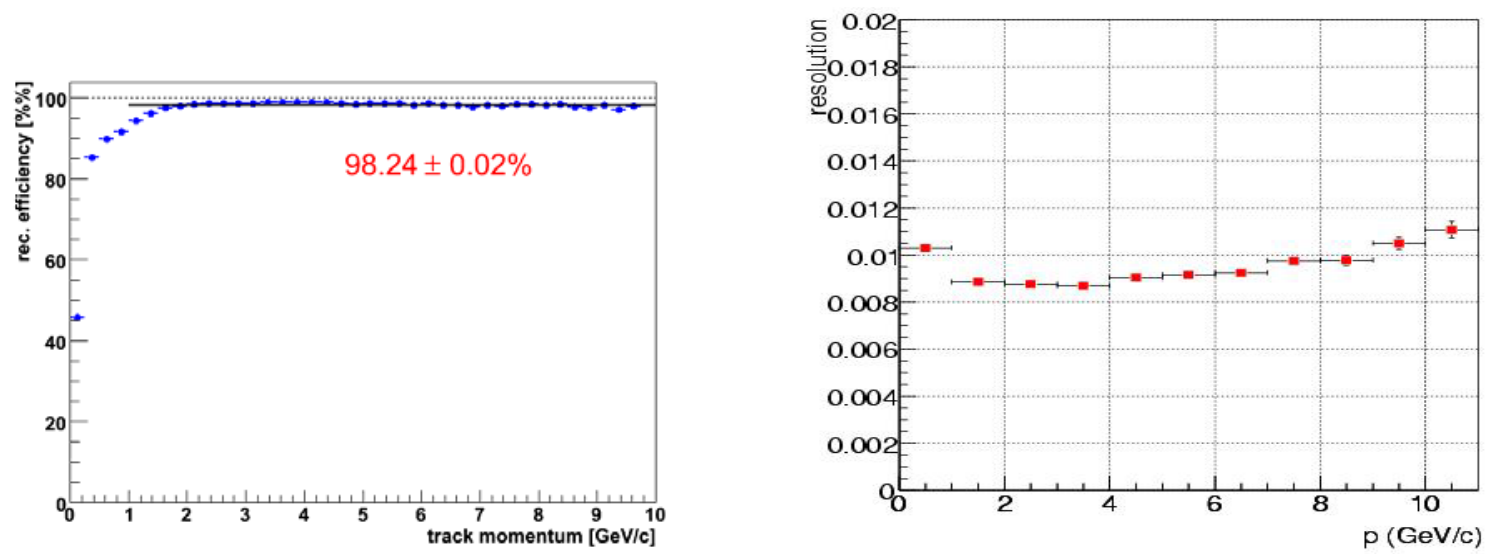

Figure 3. (Left) Reconstruction efficiency and (right) relative momentum resolution in the STS obtained for central Au+Au collisions at $25 \mathrm{AGeV}$ with the CA track finder 
at $25 \mathrm{AGeV}$ beam momentum. About $95 \%$ of all primary tracks are reconstructed. The relative momentum resolution (figure 3 right) is about $1 \%$, slightly dependent on momentum. This performance fulfills the requirements imposed by the observables to be covered by CBM.

Even without hadron identification, the measurement of hyperons via their weak decay topology is possible with STS information only, as demonstrated in figure 4. By reconstruction of the decay vertex, $\Lambda, \Xi$ and $\Omega$ baryons are detected almost background free with good acceptance and efficiency.
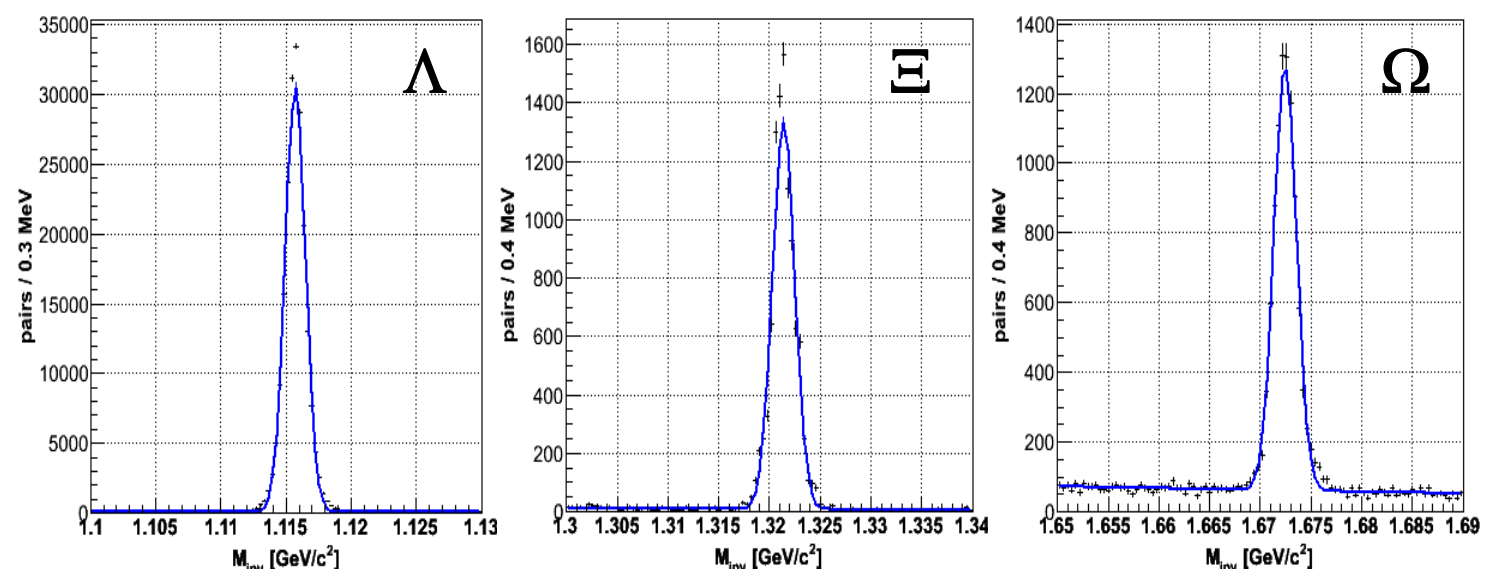

Figure 4. Invariant-mass signals for $\Lambda, \Xi$ and $\Omega$ baryons reconstructed in simulated central $A u+A u$ events at $25 \mathrm{AGeV}$

\section{MVD - the key to open charm}

At FAIR energies, charm is produced close to threshold. Consequently, the expected multiplicity of charmed mesons is extremely small - about $2 \cdot 10^{-4} \mathrm{D}^{0}$ mesons per central $\mathrm{Au}+\mathrm{Au}$ collision are predicted e. g. by the HSD model. The measurement of such rare signals via the decay into charged hadrons in a background of several hundreds of prompt charged particles per collision is one the most challenging tasks for the CBM experiment. Detection of the displaced decay vertices with excellent resolution is thus mandatory.

The micro-vertex detector of CBM will be composed of two or three ultra-thin detector layers based on monolothic active pixel sensors (MAPS) operated in vacuum close to the target. These sensor type provides a low enough material budget and sufficient coordinate resolution for high-precision vertex detection, as demonstrated in figure 5. However, both radiation hardness and read-out speed do not fulfill the CBM requirements at present. R\&D to improve on these issues is ongoing at IPHC Strasbourg. 
The obtained secondary vertex resolution of $50-60 \mu \mathrm{m}$ enables the detection of $\mathrm{D}^{0}$ (c $\tau=$ $123 \mu \mathrm{m})$ and $\mathrm{D}^{ \pm}(\mathrm{c} \tau=312 \mu \mathrm{m})$ as shown in figure 5 (right) for the latter case. The measurement of $\Lambda_{\mathrm{c}}$, recently proposed as sensitive probe for the dense medium [11] is of particular challenge due to its short life time $(60 \mu \mathrm{m})$ and surely requires the applications of latest technologies.
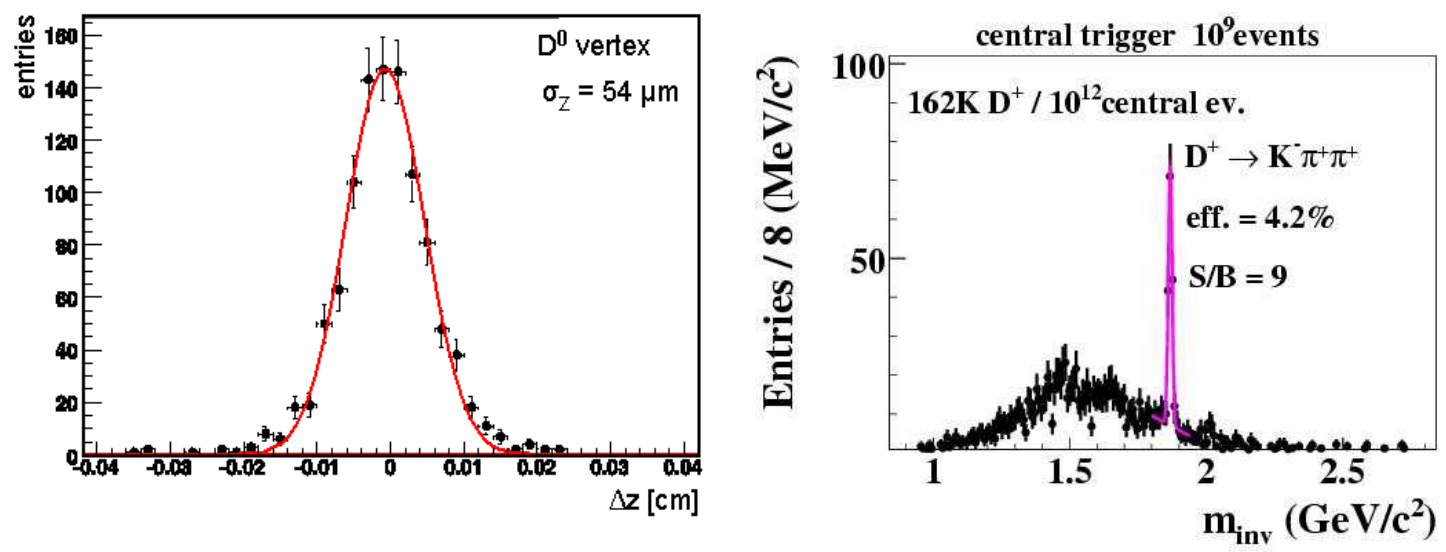

Figure 5. (Left) Distance between reconstructed and true vertex of $D^{0} \rightarrow \pi^{+} K^{-}$embedded into simulated central $\mathrm{Au}+\mathrm{Au}$ events at $25 \mathrm{AGeV}$. (Right) Reconstructed invariant-mass spectrum for the decay $D^{+} \rightarrow \pi^{+} \pi^{+} K^{-}$

\section{TOF - large acceptance hadron identification}

The identification of pions, kaons and protons emerging from close to the interaction point will be accomplished by a time-of-flight system located about $10 \mathrm{~m}$ downstream of the target. The measurement of flow and event-by-event fluctuations of particle yield ratios requires a large $(\approx 2 \pi)$ and approximately uniform acceptance; consequently, a large area of about $120 \mathrm{~m}^{2}$ must be covered. The timing resolution required for the separation of kaons and pions for momenta up to $4 \mathrm{GeV}$ is about $80 \mathrm{ps}$.

The TOF system will be realised with timing resistive plate chambers, which allow largearea coverage at moderate prices. The current design foresees a modular structure with pad read-out in the inner region and strip read-out at the periphery. The R\&D challenges are to obtain a uniform system resolution over the entire acceptance and the capability to stand the anticipated hit rates of up to $25 \mathrm{kHz} / \mathrm{cm}^{2}$. Detector development is ongoing in line with the TOF upgrades of the FOPI and HADES experiments. First results obtained from prototype tests are encouraging; no deterioration in timing resolution is seen for hit rates corresponding to the design value (figure 6 left).

Figure 6 (right) demonstrates the hadron identification capabilities of the TOF system. Kaons can be separated from pions for momenta up to $4 \mathrm{GeV}$, while protons can be identified up to $8 \mathrm{GeV}$. The acceptance for identified hadrons, shown in figure 7, covers the bulk of the production for all three particle species. 

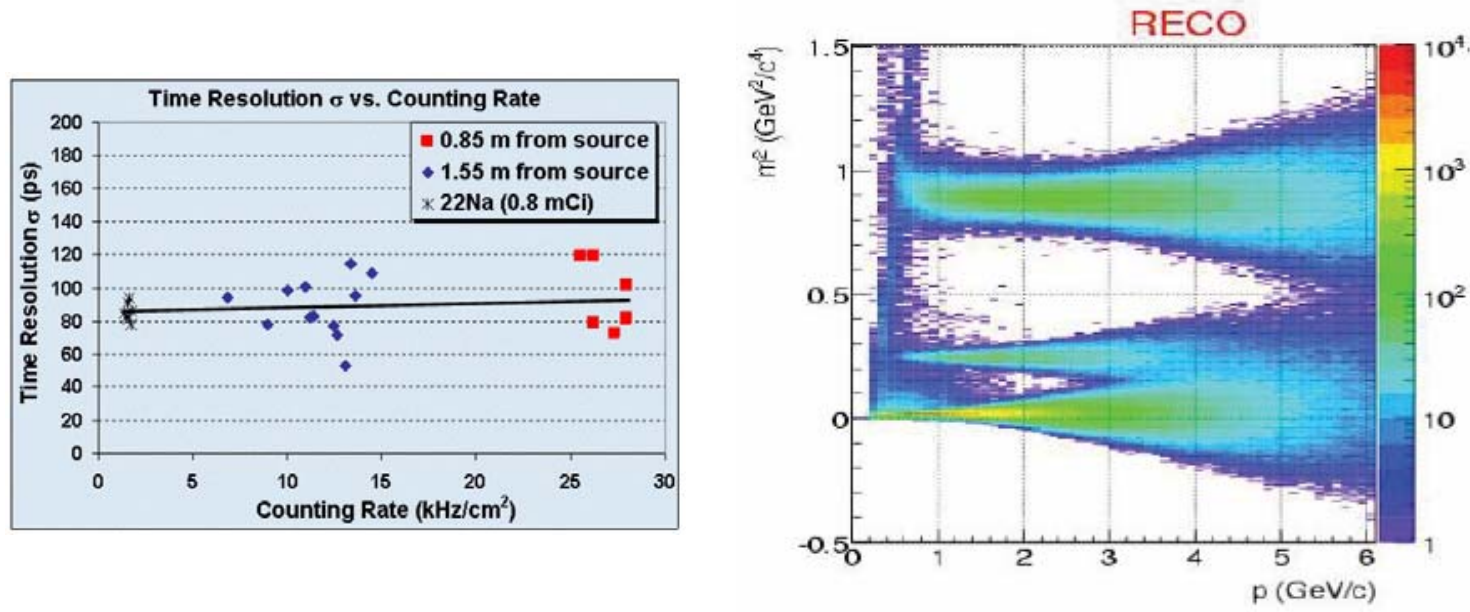

Figure 6. (Left) Time resolution of a prototype single-gap RPC as function of counting rate, obtained at LIP Coimbra. (Right) Reconstructed squared mass of primary hadrons in the TOF acceptance as function of momentum

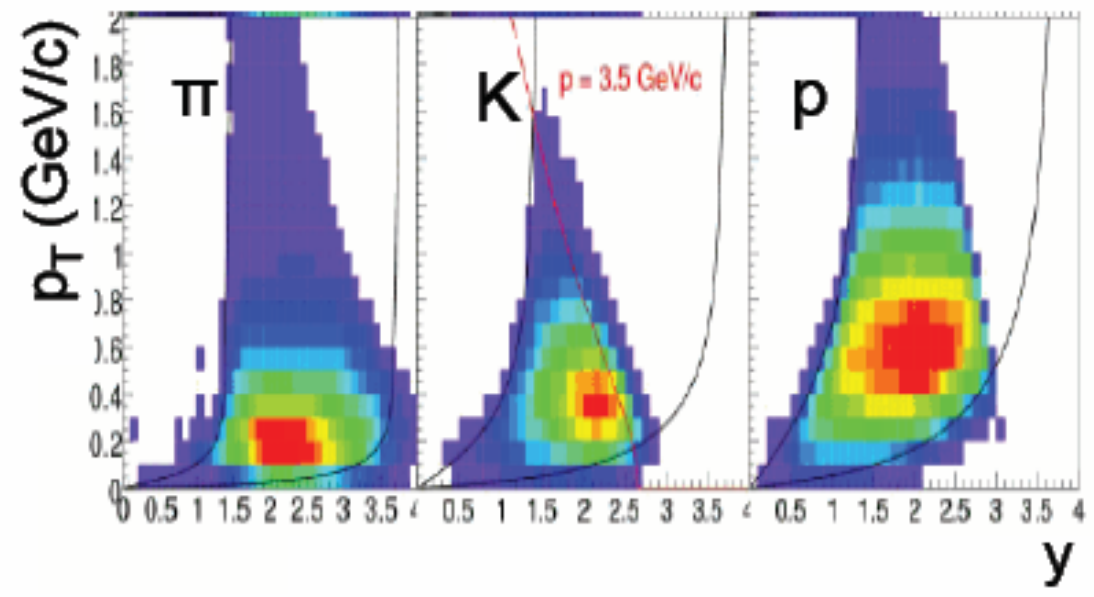

Figure 7. Distribution of TOF-identified primary hadrons in rapidity and transverse momentum in central Au+Au collisions at 25 AGeV. Mid-rapidity is 1.98 .

\section{Electron identification in RICH and TRD}

For the measurement of electron pairs CBM employs a combination of a ring-imaging cherenkov detector and several stations of transition radiation detectors. The latter also serve global tracking by connecting the tracks reconstructed in the STS with the TOF and ECAL detectors.

The RICH detector will be located directly after the STS. Cherenkov photons produced in the radiator are focussed into rings on two vertically separated planes, which are shielded from direct tracks by the magnet yokes. Figure 8 shows an event display of reconstructed rings in central $\mathrm{Au}+\mathrm{Au}$ collisions at $25 \mathrm{AGeV}$ together with the distribution of ring radius as function of momentum. Electrons can be separated from pions up to a momentum of $12 \mathrm{GeV}$. 

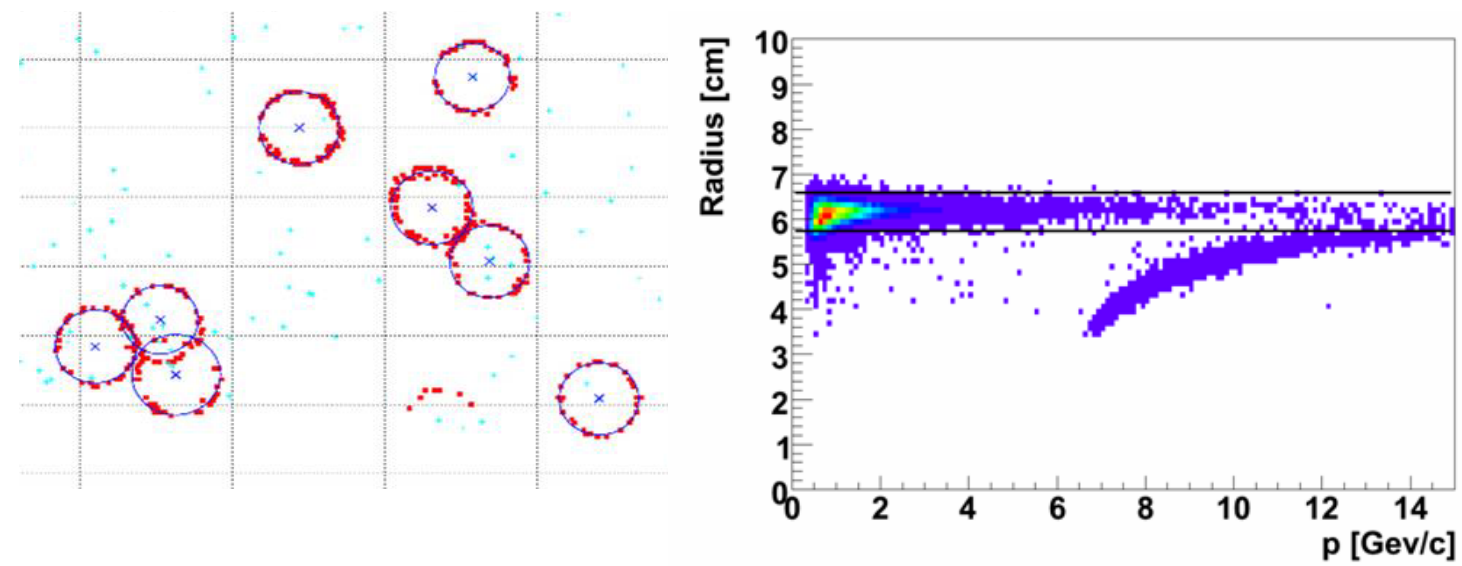

Figure 8. (Left) Reconstructed rings in the RICH photo-detector plane for central Au+Au collisions at $25 \mathrm{AGeV}$. (Right) Reconstructed ring radius as function of momentum

The TRD is composed of 12 detector layers grouped into three stations and read out by multi-wire proportional chambers. Again, the challenge is put by hit rates up to $150 \mathrm{kHz} / \mathrm{cm}^{2}$. However, thin, ALICE-like prototypes have already been tested at GSI and demonstrated gain stability up to the required count rates.

The combined performance of RICH and TRD is shown in figure 9. A pion suppression of about $10^{4}$ is reached at electron efficiencies of about $70-80 \%$ for momenta above $1 \mathrm{GeV}$. At lower momenta, the additional use of TOF information still improves on the rejection of pions.

Having rejected the vast majority of hadrons, the low-mass di-electron measurements still suffer from physical background originating from Dalitz decays of $\pi^{0}$ and $\eta$ as well as from photon conversion in the target. Unlike previous di-electron experiments, CBM identifies electrons after the magnetic field, thus providing a good invariant-mass resolution but also resulting in soft background tracks being bent out of the acceptance, leaving their partners to be contributing to the combinatorial background. Hence, a caraful rejection strategy and the usage of a thin target are mandatory. Figure 10 shows the simulated CBM performance with dielectrons for low-mass vector mesons and charmonium in central $\mathrm{Au}+\mathrm{Au}$ events at $25 \mathrm{AGeV}$
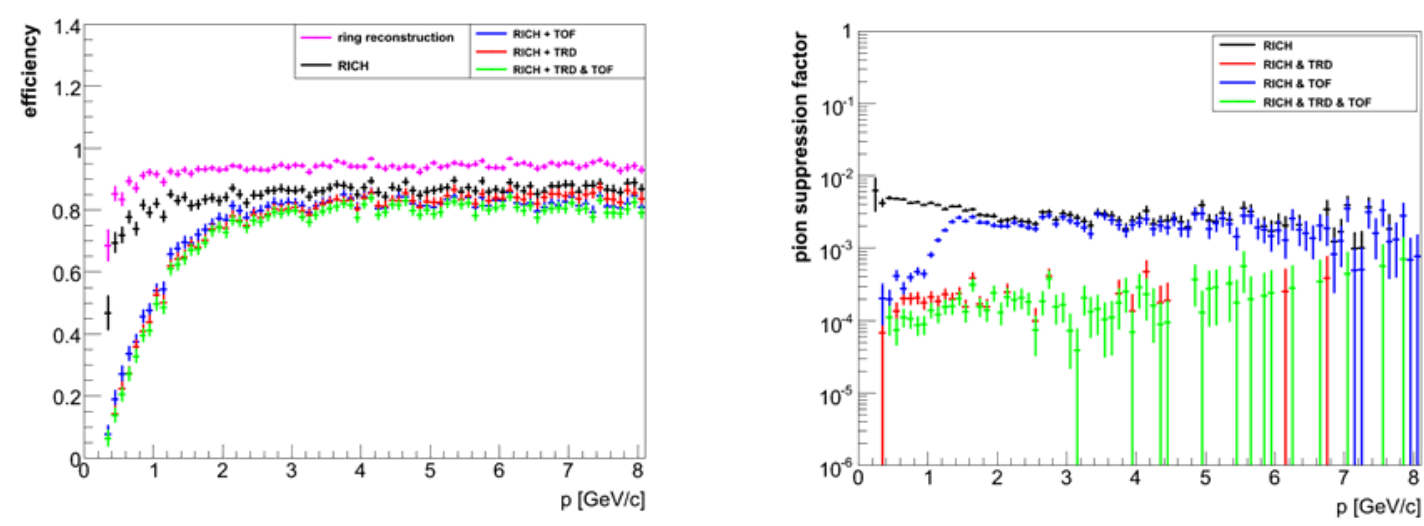

Figure 9. (Left) Electron efficiency and (right) pion suppression obtained with combined RICH, TRD and TOF information in central Au+Au collisions at $25 \mathrm{AGeV}$ 

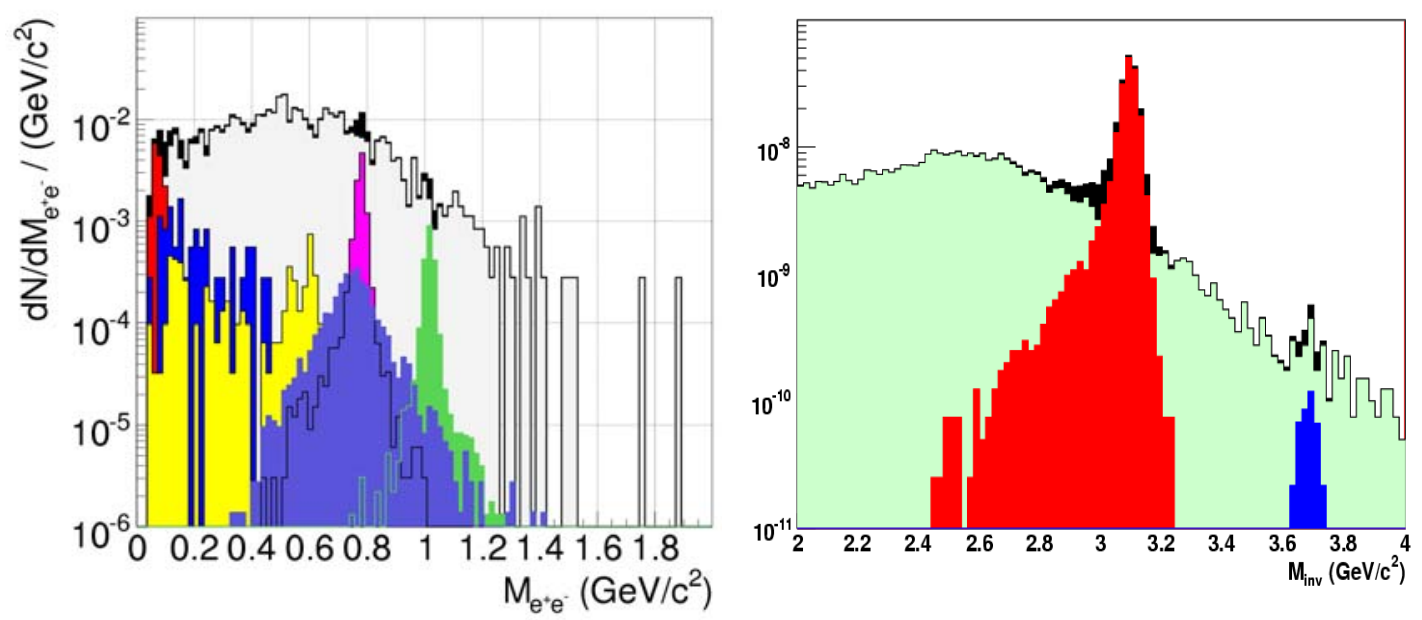

Figure 10. Invariant-mass spectra for pairs of identified electrons in the (left) low-mass vector meson region and (right) in the charmonium mass region $\left(1=J / \psi, 2=\psi^{\prime}\right)$. No transverse momentum cut was applied on the electrons from low-mass vector mesons.

beam energy, using fully reconstructed and identifed electrons. The measurement of both light and heavy vector mesons appears feasible.

\section{Di-muon measurements in the absorber system}

Alternatively to the di-electron channel, vector mesons can be detected via their decay into muon pairs. While the mass region below the two-muon threshold is not accessible by this method, it suffers less from background compared to the electron measurement, such that a trigger on muon pairs is feasible also for the low-mass part of the spectrum.

The challenge of the muon measurement is to connect muon tracks after the absorber with the abundant charged particle tracks reconstructed in the STS. CBM follows the concept of an active absorber system consisting of several GEM and/or MWPC detector stations sandwiched between iron absorber layers (figure 11). This system would replace the RICH detector in the electron setup. The outer detector system (TRD, TOF, ECAL) could serve for additional suppression of background from mis-matched tracks or, with the absorbers removed, for hadron measurements just like in the electron setup.

The simulated performance of this system is shown in figure 12 again in the mass regions of light vector mesons and of charmonia, respectively. As in the electron case, the peaks of $\omega, \varphi$ and $\mathrm{J} / \psi$ are clearly visible above the combinatorial background made up of muons from pion and kaon decay before the absorber, punch-through hadrons, and track mis-matches between STS and muon system. A comparison with figure 10 shows that the performances for both the electron and the muon setups are comparable. In both cases, even the $\psi$ ' seems to be in reach. 


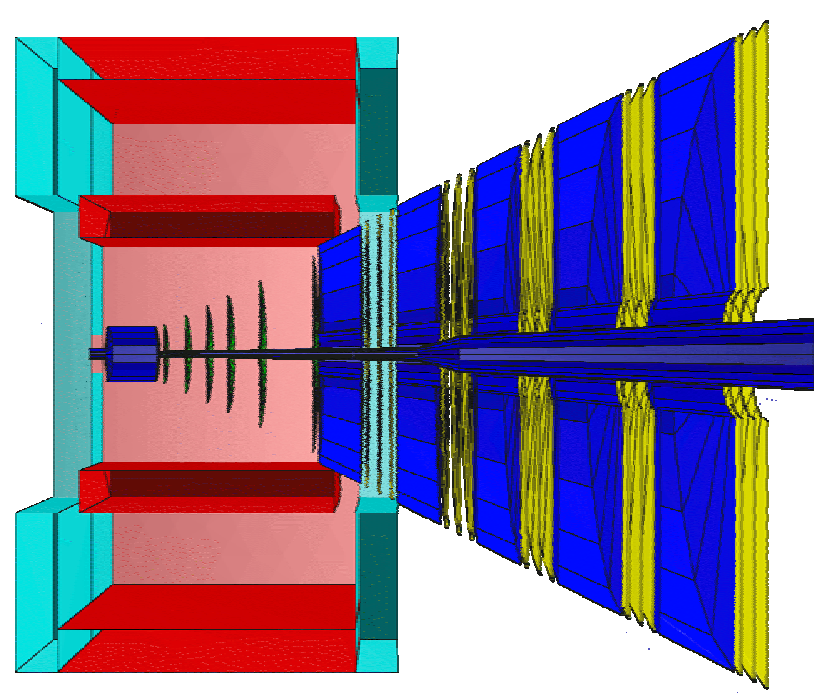

Figure 11. Sketch of the active absorber system designed for the measurement of muon pairs in CBM
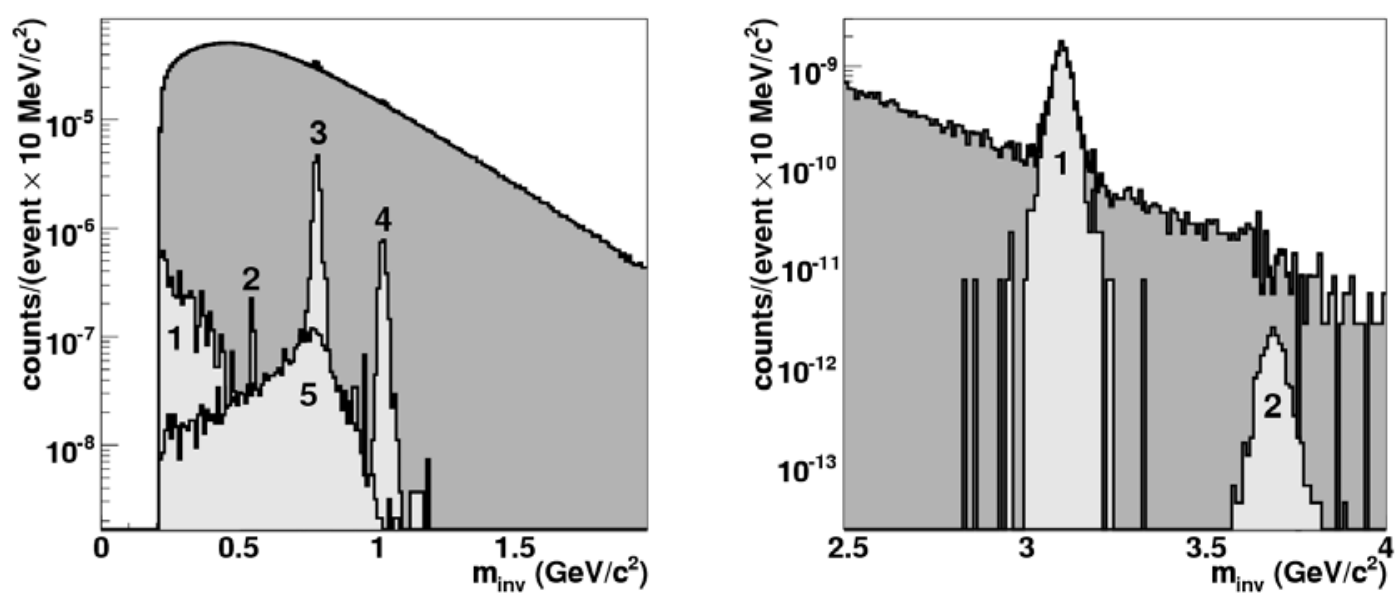

Figure 11. Invariant-mass spectra for pairs of identified muons in the (left) low-mass vector meson region $(1=$ Dalitz, $2=\eta, 3=\omega, 4=\varphi, 5=\rho)$ and (right) in the charmonium mass region $\left(1=J / \psi, 2=\psi^{\prime}\right)$

\section{Status of the experiment}

The FAIR project, first presented in a Conceptual Design Report in 2001, has meanwhile become an approved project. SIS-300 is expected to deliver beams from 2015 on.

From the beginning, the CBM experiment was considered a core experiment within the FAIR project, approved on the base of the Letter of Intent in January 2004. By today, the CBM collaboration consists of about 400 physicists from 46 institutions all over the world. The state of the preparations is laid down in the CBM Technical Status Report of 2005 [12] and, lately, the CBM Progress Report [13]. 
Performance studies leading to the final detector designs are ongoing together with detector R\&D, as briefly outlined in the previous sections, in order to reach the ambitious design specifications of CBM. The goal of these activities is to arrive at a Technical Proposal for the CBM experiment by $2011 / 2012$.

\section{References}

[1] M. Stepanow, What do we know about the QCD Phase Diagram?, these proceedings

[2] M. Gazdicki et al., J. Phys. G 31 (2004) S701

[3] Z. Fodor and S. D. Katz, Critical point of $Q C D$ at finite $T$ and $\mu$. Lattice results for finite quark masses, JHEP 0404 (2004) 050

[4] D. Cebra, RHIC low energy experiment with STAR, these proceedings

[5] K. Homma, PHENIX capabilities to probe QCD phase diagram, these proceedings

[6] A. Laszlo, NA61/NA49 future, these proceedings

[7] V. Tonneev, The NICA/MPD project at JINR, these proceedings

[8] http://wWw.gsi.de/fair/index_e.html

[9] http://WwW.gsi.de/fair/experiments/CBM/indedx_e.html

[10] S. A. Bass et al., Prog. Part. Nucl. Phys. 41 (1998) 225

[11] A. Andronic et al., arXiv:0708.1488 (2007)

[12] The CBM collaboration, CBM Technical Status Report, Darmstadt 2005, http://Www.gsi.de/documents/DOC-2005-Feb-447-1.pdf

[13] The CBM Collaboration, CBM Progress Report 2006, Darmstadt 2007, http://wWw.gsi.de/documents/DOC-2007-Mar-137-1.pdf 